\title{
Seasonal variations in nasopharyngeal carriage of respiratory pathogens in healthy Italian children attending day-care centres or schools
}

\author{
PAOLA MARCHISIO, STEFANIA GIRONI, SUSANNA ESPOSITO, GIAN CARLO SCHITO*, \\ STEFANIA MANNELLI*, NICOLA PRINCIPI and the ASCANIUS PROJECT COLLABORATIVE \\ GROUP†
}

Pediatric Department 1, University of Milan, Milan and *Institute of Microbiology; University of Genoa, Genoa, Italy

\begin{abstract}
The aim of this study was to investigate seasonal variations in the prevalence of the nasopharyngeal carriage of respiratory pathogens and identify factors affecting colonisation patterns in healthy children. The nasopharyngeal carriage of Streptococcus pneumoniae, Haemophilus influenzae and Moraxella catarrhalis during two seasons (autumn and spring) was evaluated in 1580 healthy children aged 1-7 years by means of a cohort study conducted in day-care centres and schools in eight Italian cities. A questionnaire was used to obtain the epidemiological data. In all, 309 children $(\mathbf{1 9 . 5 \% )}$ carried one or more respiratory pathogens in the autumn, and 375 children $(23.7 \%)$ in the spring. This variation was due to $H$. influenzae alone or in combination (autumn: $S$. pneumoniae 60, 3.8\%; H. influenzae 206, 13.0\%, M. catarrhalis 71, 4.5\%; spring: $S$. pneumoniae 75, 4.7\%; H. influenzae 288, 18.2\%, M. catarrhalis 82, 5.2\%). Colonisation with two or more pathogens increased from $9.1 \%$ in the spring to $17.3 \%$ in the autumn. Seasonal variations occur in the prevalence of the nasopharyngeal carriage of respiratory pathogens in healthy children attending day-care centres or schools in Italy. However, although statistically significant, the difference was slight and had limited clinical relevance. Therefore, seasonal influence on the nasopharyngeal carriage of respiratory pathogens in healthy children was negligible.
\end{abstract}

\section{Introduction}

Streptococcus pneumoniae, Haemophilus influenzae and Moraxella catarrhalis can colonise the nasopharynx and can cause upper respiratory tract infections, including otitis media and sinusitis $[1,2]$.

The prevalence of nasopharyngeal carriage of the potential pathogens varies according to age, geographic area, crowding, concomitant respiratory tract illness and antibiotic consumption [3-7]. Prospective studies have revealed considerable intra-individual quantitative and qualitative changes in nasopharyngeal flora over time [8-10]. Most studies have involved small populations, evaluated a single pathogen, were carried out in sick children, or primarily enrolled infants and young

Received 6 March 2001; revised version accepted 31 May 2001.

Corresponding author: Dr N. Principi (e-mail: Nicola. Principi@unimi.it).

$\dagger$ See Appendix. children. Variables related to the dynamics of nasopharyngeal carriage or antibiotic use have been studied extensively, but the role of seasonal variation has not been studied in a large population. Knowing the influence of season on the nasopharyngeal carriage of respiratory pathogens might be useful for epidemiological surveys. The present study investigated the influence of season on the prevalence of the nasopharyngeal carriage of respiratory pathogens in healthy Italian children and attempted to identify the factors affecting colonisation patterns.

\section{Patients and methods}

\section{Study design}

The study was part of the Italian Ascanius Project, which was designed in 1996 to evaluate the nasopharyngeal carriage of respiratory pathogens among healthy children aged 1-7 years attending day-care centres or the first 2 years of primary school [10]. Eighteen cities (10 in northern, 3 in central and 5 in southern Italy) 
participated in the study. Children with any underlying illness (including chronic otitis media with effusion), or any even mild acute upper or lower respiratory tract infection in the previous 2 weeks were excluded. The first survey was done in the autumn (mid-October to mid-November), at the beginning of the school season; the same children were then scheduled for nasopharyngeal sampling in the spring (mid-April to mid-May). To be eligible for the second nasopharyngeal culture, they had to be disease-free at the time of sampling.

Written questionnaires concerning demographics and medical history, including the number and type of respiratory infections (rhinitis, otitis media, tonsillitis, sinusitis, pneumonia) and the number and type of antimicrobial drugs administered in the 3 months preceding sampling, were completed by the parent(s) in the presence of a paediatrician. Signed informed consent was obtained from a parent or guardian of each child. Further details of the study protocol were reported in the article describing the results of the first survey [7].

\section{Specimen collection}

The nasopharyngeal specimens were obtained by the same specially trained investigator in each centre. A Mini-Culturette ${ }^{\circledR} \quad$ (Becton Dickinson, Cockeysville, MD, USA) extra-thin flexible wire swab, with the tip bent at an angle of $c .30^{\circ}$ was inserted through the child's mouth and placed 1 to 1.5 inches into the nasopharynx, without touching the uvula or the tongue. The children were not allowed to eat or drink during the $3 \mathrm{~h}$ preceding specimen collection.

\section{Microbiological procedures}

The same procedures were used by all of the participating centres, with guidelines validated by the chief microbiologist (G.C.S). The nasopharyngeal cultures were inoculated into Stuart (Venturi Transystem, Italy) transport medium tubes, which were taken to the microbiology laboratory of the hospital of each paediatric department involved within 1-3 h of collection and processed immediately. $S$. pneumoniae, $H$. influenzae and $M$. catarrhalis were isolated and identified by standard laboratory procedures. At each site, the isolates were stored in serum-glycerol freezing medium at $-80^{\circ} \mathrm{C}$; the frozen isolates were then sent to the University of Genoa's Institute of Microbiology to confirm their identity and for antimicrobial susceptibility testing. Identity was confirmed by the following procedures. H. influenzae: Gram's stain morphology, X (haemin), V (nicotinamide adenine dinucleotide) and combined XV factor dependency; S. pneumoniae: Gram's stain morphology, catalase reaction, optochin susceptibility and bile solubility; $M$. catarrhalis: Gram's stain morphology, catalase reaction and hydrolysis of tributyrin. The MICs of the antimicrobial agents were determined by the 'Sensititre microbroth' incorporation technique (Trek Diagnostic System Ltd, East Grinstead, W. Sussex) with an inoculum of $c$. $10^{5} \mathrm{cfu} / \mathrm{ml}$. The medium used was Mueller-Hinton broth, supplemented with saponin-lysed horse blood and factor $\mathrm{V}$ for isolates of Haemophilus spp. and $S$. pneumoniae. The antimicrobial agents tested were penicillin, amoxicillin, amoxicillin/clavulanate, cefaclor, cefuroxime, cefixime, ceftriaxone, erythromycin, clarithromycin and azithromycin. The MIC breakpoints used for the qualitative interpretation of the quantitative data were based on those published by the National Committee for Clinical Laboratory Standards [11].

\section{Data management and analysis}

The data were recorded on pre-coded forms and analysed using release 6.12 of the SAS program (SAS Institute, Cary, NC, USA). The quantitative variables were compared by means of Student's test and, if necessary, the qualitative variables by means of the $\chi^{2}$ test with Yates' correction or Fisher's exact test. All the reported $\mathrm{p}$ values are two-sided and refer to a significance level of $5 \%$.

\section{Results}

\section{Study participation}

Table 1 shows the demographic characteristics of the study cohort, which included the 1580 children sampled during the two seasons. Most of the children lived in an urban area, attended a day-care centre full time, and belonged to small families. None of them had received $H$. influenzae $\mathrm{b}$ vaccine. There was no significant difference between the children sampled in the autumn and spring in terms of family size, living conditions, full-time attendance at a day-care centre or passive smoking exposure. The cohort consisted of $91.7 \%$ of the 1723 children enrolled in the autumn survey because 143 children did not return for the spring sampling session due to the following reasons: moved outside the study area $(n=65)$, changed school $(\mathrm{n}=25)$, were unwilling to be sampled again $(\mathrm{n}=15)$ or developed a chronic illness $(n=38)$.

Table 1. Characteristics of the study cohort

\begin{tabular}{lr}
\hline Characteristic & $\begin{array}{r}\text { Number of children } \\
((\%)=1580)\end{array}$ \\
\hline Male & $815(51.6)$ \\
Race (white) & $1552(98.2)$ \\
Age (mean) & 4.39 \\
$\quad 1-3$ years & $611(38.7)$ \\
$4-5$ years & $390(24.7)$ \\
$6-7$ years & $579(36.6)$ \\
Breast-feeding $\geqslant 3$ months & $1166(73.8)$ \\
Living in urban area (population: $>10000)$ & $1270(80.4)$ \\
Full-time day-care attendance* & $1478(93.5)$ \\
Previous day-care attendance $(>1$ year) & $853(53.9)$ \\
Having older sibling(s) & $972(61.5)$ \\
Living in a small family $(3-4$ people) & $1446(91.5)$ \\
Passive smoking & $750(47.5)$ \\
\hline
\end{tabular}

*Attendance for 5-6 days/week, 6-8 h/day. 
Prevalence of nasopharyngeal carriage of respiratory pathogens in the two seasons

Table 2 shows the nasopharyngeal colonisation of respiratory pathogens in the two seasons. In all, 309 children $(19.5 \%)$ carried respiratory pathogens in the autumn, compared with $375(23.7 \%)$ in the spring $(p=0.004)$. The proportion of carriers colonised by two or three pathogens increased significantly between the autumn (28 of $309,9.1 \%)$ and spring (65 of $375,17.3 \%)(\mathrm{p}=0.002)$

The carriage of $H$. influenzae, alone or in combination with other pathogens, was significantly more common in the spring than in the autumn $(\mathrm{p}=0.02$ and $\mathrm{p}=0.0001$, respectively). No significant difference in the carriage rates of $S$. pneumoniae or M. catarrhalis was found between the two surveys.

\section{Antibiotic susceptibility patterns}

The susceptibility patterns of the respiratory pathogens in the two surveys were not significantly different. Among the $S$. pneumoniae isolates, only a few isolates were penicillin resistant - autumn: three isolates $(5 \%)$, two intermediately resistant and one fully resistant; spring: four isolates $(5.3 \%)$, three intermediately resistant, one fully resistant - whereas resistance to macrolides was very common - autumn: 25 isolates $(41.6 \%), 1$ intermediately resistant, MIC $\geqslant 0.5 \mathrm{mg} / \mathrm{L}$, 24 fully resistant, MIC $\geqslant 1 \mathrm{mg} / \mathrm{L}$; spring: 30 isolates (40\%), 2 intermediately resistant, 28 fully resistant. All S. pneumoniae isolates were susceptible to amoxicillin, independently of the season. Resistance to amoxicillin remained stable for the $M$. catarrhalis isolates autumn: 63 isolates (88.7\%); spring: 73 isolates, $89.1 \%$ - and increased slightly and in a non-significant manner in the isolates of $H$. influenzae - autumn: 12 isolates (5.8\%); spring: 29 isolates, $(10.1 \%)$.

\section{Variables associated with differences in nasopharyngeal carriage in the two seasons}

Table 3 summarises the association between nasopharyngeal colonisation and respiratory tract infections or antibiotic courses in the 3 months preceding each sampling session. The overall proportion of children who had experienced respiratory tract infections or had been given antibiotics was greater in the spring than in the autumn, but there was no difference in these variables between the carriers of one or more pathogens and the non-carriers in each study period. The drug types used were similar in both periods.

The proportion of children that had two or more respiratory tract infections in the preceding 3 months was significantly higher in the spring than in the autumn only in the subjects carrying $H$. influenzae autumn: 31 of 206 (15.0\%); spring: 67 of 288 (23.3\%); $\mathrm{p}=0.03$ - and children carrying two or more pathogens - autumn: 3 of 28 (10.7\%); spring: 22 of 65 $(33.8 \%) ; p=0.04$. No significant difference was found between the autumn and spring among the carriers of H. influenzae or multiple pathogens, or in the use or type of antibiotics. No other significant differences were found.

\section{Discussion}

The data from the present study seem to indicate that the prevalence of nasopharyngeal carriage of respiratory pathogens can vary over time, with a small but significant increase from autumn to spring, in healthy children attending day-care centres or schools. $H$. influenzae, alone or in combination, was the main organism responsible for this increase. However, although statistically significant, the increase in the proportion of children who were asymptomatic nasopharyngeal carriers of respiratory pathogens between the autumn and spring was slight and of limited clinical relevance.

These findings differ from those of the limited number of reports involving infants and children followed over time. Harvey and Dunlap [3] did not find any consistent seasonal fluctuations in the nasal carriage of $S$. pneumoniae, $H$. influenzae or haemolytic

Table 2. Nasopharyngeal colonisation of respiratory pathogens in the autumn and spring

\begin{tabular}{lccc}
\hline & \multicolumn{2}{c}{ Number $(\%)$ of children (total $=1580)$} \\
\cline { 2 - 4 } Carrier status & Autumn & Spring & p value \\
\hline Carriers of one or more pathogens & $309(19.5)$ & $375(23.7)$ & 0.004 \\
$S$. pneumoniae & $60(3.8)$ & $75(4.7)$ & 0.23 \\
$H$. influenzae & $206(13.0)$ & $288(18.2)$ & $<0.0001$ \\
M. catarrhalis & $71(4.5)$ & $82(5.2)$ & 0.40 \\
Carriers of one pathogen & $281(17.8)$ & $310(19.6)$ & 0.20 \\
$S$. pneumoniae & $47(2.9)$ & $39(2.5)$ & 0.44 \\
$H$. influenzae & $185(11.7)$ & $228(14.4)$ & 0.02 \\
$M$. catarrhalis & $49(3.1)$ & $43(2.7)$ & 0.59 \\
Carriers of two pathogens & $28(1.8)$ & $60(3.8)$ & 0.0008 \\
$S$. pneumoniae $+H$. influenzae & $6(0.3)$ & $26(1.6)$ & 0.0007 \\
$S$. pneumoniae $+M$. catarrhalis & $7(0.4)$ & $5(0.3)$ & 0.77 \\
$H$. influenzae $+M$. catarrhalis & $15(0.9)$ & $29(1.8)$ & 0.04 \\
Carriers of three pathogens & 0 & $5(0.3)$ & 0.06 \\
\hline
\end{tabular}


Table 3. Association between respiratory tract infections and antibiotic prescriptions in the preceding 3 months and nasopharyngeal colonisation in autumn and spring

\begin{tabular}{|c|c|c|c|c|c|c|}
\hline \multirow[b]{3}{*}{ Parameter } & \multicolumn{6}{|c|}{ Number $(\%)$ of children } \\
\hline & \multicolumn{3}{|c|}{ Autumn } & \multicolumn{3}{|c|}{ Spring } \\
\hline & $\begin{array}{c}\text { Total } \\
(\mathrm{n}=1580)\end{array}$ & $\begin{array}{l}\text { Carriers } \\
(\mathrm{n}=309)\end{array}$ & $\begin{array}{l}\text { Non-carriers } \\
(\mathrm{n}=1271)\end{array}$ & $\begin{array}{c}\text { Total } \\
(\mathrm{n}=1580)\end{array}$ & $\begin{array}{l}\text { Carriers } \\
(\mathrm{n}=375)\end{array}$ & $\begin{array}{c}\text { Non-carriers } \\
(\mathrm{n}=1205)\end{array}$ \\
\hline \multicolumn{7}{|c|}{ Respiratory tract infections: } \\
\hline one & $547(34.6)$ & $100(32.4)$ & $447(35.1)$ & $596(37.8)$ & $137(36.5)$ & $459(38.1)$ \\
\hline two or more & $292(18.5)$ & $60(19.4)$ & $232(18.3)$ & $315(19.9)$ & $70(18.7)$ & $245(20.3)$ \\
\hline Antibiotic therapy* & $427(27.0)^{*}$ & $81(26.2)^{\S}$ & $346(27.2)^{\|}$ & $503(31.8)$ & $126(33.6)$ & $377(31.2)$ \\
\hline Aminopenicillins & $110(6.9)$ & $21(6.8)$ & $89(7.0)$ & $133(8.4)$ & $32(8.5)$ & $101(8.4)$ \\
\hline Amoxicillin-clavulanate & $33(2.1)$ & $4(1.3)$ & $29(2.3)$ & $50(3.2)$ & $12(3.2)$ & $38(3.2)$ \\
\hline Macrolides & $140(8.8)$ & $28(9.1)$ & $112(8.8)$ & $145(9.2)$ & $37(9.9)$ & $108(8.9)$ \\
\hline Cephalosporins & $142(8.9)$ & $28(9.1)$ & $114(8.9)$ & $175(11.1)$ & $45(12.0)$ & $130(10.8)$ \\
\hline
\end{tabular}

* One or more courses of antibiotics.

Autumn versus spring: ${ }^{\dagger} \mathrm{p}=0.01 ;{ }^{\ddagger} \mathrm{p}=0.003 ;{ }^{\S} \mathrm{p}=0.04 ;{ }^{\|} \mathrm{p}=0.02$. The differences between carriers and non-carriers were not significant in either study period.

streptococci in 97 family groups. More recently, Ingvarsson et al. [4] observed that the colonisation rate of respiratory pathogens decreased from $69 \%$ to $55 \%$ in healthy children between November and April, without any difference in the distribution of individual bacteria. Furthermore, increased carriage of $S$. pneumoniae in the winter has been described by Gray et al. [12]. Brook and Gober [13] observed an increase in the carriage of $\beta$-lactamase-producing bacteria between the autumn and spring in children with acute pharyngotonsillitis.

Possible explanations for the discrepancies between the data from the latter studies and the present study include the following. First, the large size of the present cohort study population was more representative of the enrolled age groups and geographic sites, and may have avoided the risk of focusing on specific situations. Secondly, the longitudinal follow-up of the same cohort assured a better description of seasonal variation than that obtained from random samples (with different populations). Thirdly, the cohort in the present study included only healthy children who, even if attending day-care centres or schools, had rarely experienced upper respiratory tract infection within the 3 months preceding both samplings. This excluded the possible effect of concomitant viral infections or mild underlying diseases such as otitis media with effusion on nasopharyngeal carriage $[5,13]$, and may also account for the low prevalence of carriage in both sessions. Moreover, in the two observational periods there were no epidemic conditions that might have affected the results.

The present study had two limitations: seasonal variations would have been better described if there were more sampling points or if observations had been repeated in other years, and the lack of biotyping, which would have allowed a better understanding of the dynamics of the individual pathogens. However, the main purpose of the study was not to assess the dynamics of a specific organism, but to evaluate the influence of season on the prevalence of the nasopharyngeal carriage of respiratory pathogens in healthy children attending child-care centres.

Respiratory tract infections in the preceding 3 months were not associated with the carriage of any of the respiratory pathogens in the autumn [7], but a history of two or more episodes of upper respiratory tract infection was the only variable associated with the higher carriage rate of $H$. influenzae in the spring. As most upper respiratory tract infections occurring in the winter are caused by viruses, the increase in the carriage rate of $H$. influenzae in the spring might be explained on the basis of data derived from in-vitro studies, which showed that respiratory viruses (particularly adenovirus) can enhance bacterial adherence to the nasopharynx and thus influence nasopharyngeal carriage $[14,15]$.

The susceptibility patterns of the isolates studied did not change significantly over time. The rates of penicillin resistance among $S$. pneumoniae and $\beta$ lactamase production among $H$. influenzae isolates were always very low, and consistent with previous Italian reports regarding adults and children with respiratory tract infections $[10,16,17]$. In a child-care centre, Boken et al. [18] recently observed a significant reduction from $53 \%$ to $7 \%$ in penicillin-resistant $S$. pneumoniae colonisation over a 4-month period, concomitant with a marked decrease in antibiotic use. Furthermore, Brook and Gober [13] have suggested that the decrease from winter to summer in the recovery rate of $\beta$-lactamase-producing bacteria could be the result of a different exposure to $\beta$-lactams in the two seasons. In the present study, the slight increase in antibiotic consumption between autumn and spring may have prevented the seasonal reduction described in other studies, whereas the similar distribution of the 
type of drugs used in the 3 months preceding each sampling may have avoided the selective colonisation of resistant isolates.

In conclusion, if the data are analysed with regard to their importance in planning collective surveys of nasopharyngeal carriage in healthy children attending day-care centres or schools [19], the slight increase in carriage from autumn to spring seems to be of limited practical value, and suggests that such surveys can be undertaken without regard to season.

\section{Appendix}

The Ascanius Project Collaborative Group includes: E. Reali (Cinisello Balsamo), R. Longhi (Como). G.L. Marseglia (Pavia), D. Pavesio (Torino), G. Bona (Novara), B. Sacher (S. Daniele Friuli), M. De Santis (Genova), G.P. Salvioli (Bologna), G. Dodi (Fidenza), G. Farneti (Cesena), G. Caramia (Ancona), P. Baroncelli (Pisa), T. Meloni (Sassari), A. Guarino (Napoli), A. Russo (Bari), F. Pusceddu (Cagliari), L. Ziino (Palermo), D.C. Salpietro (Messina).

This work was supported in part by an educational grant from SmithKline Beecham Pharmaceuticals, Italy.

\section{References}

1. Todd JK. Bacteriology and clinical relevance of nasopharyngeal and oropharyngeal cultures. Pediatr Infect Dis J 1984; 3: $159-163$.

2. Faden H, Duffy L, Wasielewski R et al. Relationship between nasopharyngeal colonization and the development of otitis media in children. $J$ Infect Dis 1997; 175: 1440-1445.

3. Harvey HS, Dunlap MB. Seasonal prevalence of upper respiratory pathogens. $N$ Engl $J$ Med 1961; 264: 684-686.

4. Ingvarsson L, Lundgren K, Ursing J. The bacterial flora in the nasopharynx in healthy children. Acta Otolaryngol 1982; 94 Suppl 386: 94-96.

5. Faden H, Waz MJ, Bernstein JM, Brodsky L, Stanievich J, Ogra PL. Nasopharyngeal flora in the first three years of life in normal and otitis-prone children. Ann Otol Rhinol Laryngol 1991; 100: 612-615.
6. Soewignjo S, Gessner BD, Sutanto A et al. Streptococcus pneumoniae nasopharyngeal carriage prevalence, serotype distribution, and resistance patterns among children on Lombok island, Indonesia. Clin Infect Dis 2001; 32: 1039-1043.

7. Principi N, Marchisio P, Schito GC, Mannelli S and the Ascanius Project Collaborative Group. Risk factors for carriage of respiratory pathogens in the nasopharynx of healthy children. Pediatr Infect Dis J 1999; 18: 517-523.

8. Faden H, Stanievich J, Brodsky L, Bernstein J, Ogra PL. Changes in nasopharyngeal flora during otitis media of childhood. Pediatr Infect Dis J 1990; 9: 623-626.

9. Cohen R, Bingen E, Varon E et al. Change in nasopharyngeal carriage of Streptococcus pneumoniae resulting from antibiotic therapy for acute otitis media in children. Pediatr Infect Dis $J$ 1997; 16: $555-560$.

10. Ghaffar F, Friedland IR, McCracken GH. Dynamics of nasopharyngeal colonization by Streptococcus pneumoniae. Pediatr Infect Dis J 1999; 18: 638-646.

11. National Committee for Clinical Laboratory Standards. Performance standards for antimicrobial susceptibility testing; 11th information supplement. Ne CLS 2001; M100-S11, vol. 21, no. 1.

12. Gray BM, Converse GM, Dillon HC. Epidemiologic studies of Streptococcus pneumoniae in infants: acquisition, carriage, and infection during the first 24 months of life. J Infect Dis 1980; 142: $923-933$.

13. Brook I, Gober AE. Monthly changes in the rate of recovery of penicillin-resistant organisms from children. Pediatr Infect Dis $J$ 1997; 16: 255-257.

14. Håkansson A, Carlstedt I, Davies J, Mossberg A-K, Sabharwal $\mathrm{H}$, Svanborg C. Aspects on the interaction of Streptococcus pneumoniae and Haemophilus influenzae with human respiratory tract mucosa. Am J Respir Crit Care Med 1996; 154: S187-S191.

15. Miyamoto N, Bakaletz LO. Kinetics of the ascension of NTHi from the nasopharynx to the middle ear coincident with adenovirus-induced compromise in the chinchilla. Microb Pathog 1997; 23: 119-126.

16. Marchese A, Debbia E, Pesce A, Schito GC. Comparative activities of amoxycillin and 10 other drugs against penicillinsusceptible and -resistant Streptococcus pneumoniae isolates recently isolated in Italy. Clin Microbiol Infect 1998; 4: $170-173$.

17. Nicoletti G, Aleo G, Blandino $G$ et al. Osservatorio epidemiologico italiano:analisi del $2^{\circ}$ anno di monitoraggio della resistenze agli antibiotici in $H$. influenzae e $H$. parainfluenzae. GIMMOC 1999; 3: 58-69.

18. Boken DJ, Chartrand SA, Goering RV, Kruger R, Harrison CJ Colonization with penicillin-resistant Streptococcus pneumoniae in a child-care center. Pediatr Infect Dis J 1995; 14: 879-884.

19. Kellner JD, McGeer A, Cetron MS et al. The use of Streptococcus pneumoniae nasopharyngeal isolates from healthy children to predict features of invasive disease. Pediatr Infect Dis J 1998; 17: 279-286. 